La revue La revue pour l'histoire du CNRS

POUR L'HISTOIRE DU CNRS

14 | 2006

Le patrimoine scientifique

\title{
Faire du patrimoine industriel un métier
}

Le master 2 « Pist » de l'université d'Artois

Marie-Noëlle Polino et Denis Varaschin

\section{CpenEdition}

Journals

Édition électronique

URL : https://journals.openedition.org/histoire-cnrs/1772

DOI : 10.4000/histoire-cnrs. 1772

ISSN : 1955-2408

Éditeur

CNRS Éditions

Édition imprimée

Date de publication : 3 mai 2006

ISBN : 978-2-271-06350-2

ISSN : $1298-9800$

Référence électronique

Marie-Noëlle Polino et Denis Varaschin, « Faire du patrimoine industriel un métier », La revue pour I'histoire du CNRS [En ligne], 14 | 2006, mis en ligne le 03 mai 2008, consulté le 20 mai 2021. URL http://journals.openedition.org/histoire-cnrs/1772 ; DOI : https://doi.org/10.4000/histoire-cnrs.1772

Ce document a été généré automatiquement le 20 mai 2021

Comité pour l'histoire du CNRS 


\title{
Faire du patrimoine industriel un métier
}

\author{
Le master 2 « Pist » de l'université d'Artois
}

Marie-Noëlle Polino et Denis Varaschin

1 Le patrimoine correspond désormais à un secteur professionnel à part entière. Multipolaire et pluridisciplinaire, l'université d'Artois, qui possédait déjà une expérience d'enseignement professionnalisant avec un IUP " Patrimoine et tourisme ", l'a développée dans ses offres de formation en proposant en septembre 2002 sur son campus d'Arras le DESS «Mise en valeur et gestion du patrimoine technique et industriel ». Depuis septembre 2004, la réforme universitaire et le retour d'expérience des premières années d'existence ont conduit à faire sensiblement évoluer le contenu de ce diplôme, devenu master 2 professionnel Pist (Patrimoine industriel, scientifique et technique). Nous en présentons ici le champ d'action et l'implantation régionale, les orientations et les contenus, y compris les développements dans les prolongements de la « recherche-action $»^{1}$.

2 Lorsqu'il s'est agi de définir un domaine à prospecter, plusieurs éléments ont plaidé en faveur du champ industriel dans un Nord - Pas-de-Calais passé de l'artisanat et du commerce à la grande industrie au XIX ${ }^{\mathrm{e}}$ siècle. L'histoire a laissé des traces anciennes et omniprésentes: textile, charbon, industries lourdes (métallurgie, sidérurgie, chimie), agro-alimentaire à l'intérieur des terres et activité portuaire sur le littoral ont structuré la région et imprimé leurs marques au paysage. Après le repli de certaines de ces branches, le Nord-Pas-de-Calais a connu une deuxième transition fondamentale au cours des trente dernières années pour se transformer en une " eurorégion » tertiaire qui affiche la volonté d'assumer son héritage en mettant en valeur un patrimoine industriel de premier ordre ${ }^{2}$. Des témoignages symboliques destinés à permettre une médiation entre passé et présent ont été préservés puis valorisés, les collectivités territoriales voyant dans cette démarche un élément de la restructuration/ reconversion menée. Du côté des acteurs privés, le Nord-Pas-de-Calais est aujourd'hui la première région française par le nombre d'entreprises participant au tourisme de découverte économique et de visiteurs accueillis ${ }^{3}$. 
3 La mine a légué un patrimoine important : chevalements (comme Marles-les-Mines, le rescapé de la Première Guerre mondiale), terrils (Rieuley) souvent reliés entre eux par les cavaliers qui supportaient les voies ferrées du réseau des Houillères, logements qui vont du coron aux barreaux puis aux cités, anciens grands bureaux reconvertis en lieux publics (ceux des mines de Lens accueillent le pôle scientifique de l'université d'Artois). Le Centre historique minier de Lewarde, ouvert au public en 1984, domine un essaim de petits musées de la mine. Les carreaux de Wallers-Arenberg et Oignies ont été classés monuments historiques en 1992 et 1994, ce dernier accueillant la Mission du bassin minier (1998) et développant un projet de valorisation sur le thème des "sécurités industrielles ». L'Écopôle de Loos-en-Gohelle conçoit son action autour de trois axes : artistique, culturel et social ( culture commune »), naturel et écologique (" la chaîne des terrils »), sportif et de détente (à l'instar du site très couru de Nœux-les-Mines, Loisinord, dont la base nautique et la piste de ski artificielle correspondent à des terrils réaménagés). À l'occasion de la commémoration du centenaire de la catastrophe dite de Courrières (10 mars 1906), les communes de Billy-Montigny et de Méricourt inaugureront un musée en plein air ("le parcours des rescapés») et un Centre d'interprétation des risques industriels (Ciri). La communauté d'agglomération de LensLiévin réfléchit à la prochaine mise en valeur de la Maison syndicale de Lens. Parfois soucieux de vente directe, le textile offre ses logements (courées de Lille et surtout de Roubaix), ses usines (Roubaix : filature de coton Motte-Bossut occupée par le Centre des archives du monde du travail (CAMT), bâtiment du conditionnement public, usines Roussel, Dillies frères, Lepoultre réemployées à des titres divers ; Lille : filature Le Blan) et ses musées (du tissage Craye à Roubaix; de la dentelle à Calais, Bailleul et Caudry ; de la broderie à Villers-Outréaux; écomusée du textile et de la vie sociale dans l'ancienne filature Prouvost-Masurel de Fourmies; galerie du lin à Lille...). Arc International, à Arques, se pose en plus important site de tourisme d'entreprise au nord de Paris.

L'agro-alimentaire a laissé des traces nombreuses et parfois spectaculaires tant dans les campagnes (moulins, sucreries de betteraves...) que dans les villes : grands moulins de Marquette-lès-Lille, brasserie Motte-Cordonnier (Armentières) en passe de devenir un pôle patrimonial, ou plus modestes confiserie Afchain (Cambrai), musée de la chicorée d'Orchies, distillerie Claeyssens (Wambrechies) et brasserie La Choulette (Hordain). Après le Conservatoire du patrimoine rural de Villeneuve-d'Ascq, un Centre de culture scientifique et technique et industriel consacré à l'agro-alimentaire et à l'environnement, Cité Nature, a ouvert ses portes à Arras en 2004. Les transports (ascenseur hydraulique à bateaux des Fontinettes, musée portuaire de Dunkerque, sites ferroviaires) et l'énergie (des multiples moulins à l'unique centrale électronucléaire de Gravelines) complètent le tableau.

5 Enfin, le musée industriel et commercial de Lille, fondé en 1853 à l'initiative de la Société des sciences, des arts et de l'industrie de cette ville et rénové au cours des années 1990, participe à cette dynamique. Pays devenu « noir » avec l'industrialisation et en passe de redevenir " vert », le Nord - Pas-de-Calais, entre rejet de la " purification territoriale » et de la "fossilisation du territoire ", offre, avec ses réussites et ses échecs, la vision d'une région dans laquelle une volonté de conservation d'une mémoire identitaire, un souci de ne pas briser des liens esthétiques et culturels ainsi qu'une appréhension des intérêts économiques du tourisme industriel émergent plus nettement qu'ailleurs. 
6 Si la sauvegarde du patrimoine industriel est restée longtemps, en particulier dans le domaine du patrimoine minier, à l'initiative de l'État qui a dû dans les années 1990 user de la protection administrative comme d'une arme pour arrêter des destructions, aujourd'hui les collectivités publiques sont présentes dans sa mise en valeur. La plupart des exemples cités font l'objet de projets portés par les municipalités et, plus souvent, les groupements de communes, au premier rang quand il s'agit de donner à un site une fonction culturelle par la création d'un nouvel équipement (musée, centre d'information, CCSTI), dans tous les cas comme accompagnateur bienveillant des reconversions à des fins privées (logements ou bureaux). La question de la conservation pour de nouveaux usages des sites industriels urbains est aujourd'hui toujours posée, quelle que soit la réponse qui lui est donnée après des périodes de latence des projets qui peuvent atteindre la décennie. Les universités sont souvent associées aux réflexions préalables ou à des opérations d'envergure régionale comme la candidature du bassin minier à l'inscription sur la liste du patrimoine mondial, élaborée de 2003 à 2005 en incluant des dimensions de mobilisation sociale mais aussi d'étude et d'inventaire du patrimoine minier.

7 Pour l'université d'Artois, restait à mener une réflexion afin de mettre en perspective ce nouveau paysage. Sa faculté d'histoire possédait des atouts pour y participer, notamment le laboratoire consacré à l'histoire contemporaine (EA 2466), qui rassemble des chercheurs sensibles aux problématiques de l'industrialisation et de la patrimonialisation. Ils ont perçu dans le patrimoine industriel et ses enjeux politiques, économiques et culturels un créneau de formation et un domaine d'activités jusqu'alors peu exploité, à travers ses soucis et ses exigences, ses partenaires et ses métiers, dans une région qui se prêtait pourtant à sa mise en valeur. L'analyse du domaine du Pist a montré qu'il se trouve dans une phase d'élaboration, de structuration et de reconnaissance, avec la sauvegarde par les collectivités publiques de sites industriels désaffectés à des fins historiques et culturelles ou la conversion de bâtiments industriels et, pour les muséums et musées scientifiques et techniques, dans une phase de transformation et de nouveaux projets. L'enseignement proposé à Arras repose donc sur la volonté de former de véritables "généralistes ", des " pluri-spécialistes ", car ce patrimoine est un thème pluridisciplinaire, ou plutôt qui dicte le recours à des disciplines variées pour être compris dans sa totalité, quels que soient le cas traité et l'échelle à laquelle on l'envisage.

8 Quant à la notion d'interprétation du patrimoine chère au Cilac (Comité d'information et de liaison pour l'archéologie, l'étude et la mise en valeur du patrimoine industriel), que met en avant la formation, elle sous-entend des connaissances et un projet dépassant ce que l'on appelle communément «médiation». Si le patrimoine correspond à ce qui doit être transmis, s'il est le résultat d'un choix collectif et durable qui transcende l'individu, il est le fruit d'une sélection, d'une volonté pensée de transmission, de choix motivés, fussent-ils douloureux. Mais, surtout, le patrimoine implique un public présent et des publics à venir. De ce fait, il appartient aux sphères de la mémoire et de la culture commune. Le professionnel doit savoir les analyser pour reconnaître le message transmis via l'intérêt porté par la population au patrimoine dans toutes ses dimensions: devant, par exemple, une technique disparue, il faut simultanément déceler la valeur qu'on lui attache aujourd'hui et anticiper les valeurs dont elle sera investie, garantes de l'intérêt collectif, donc de la pérennité de ce patrimoine. 
9 Pour réussir cette interprétation, au sens large, et tout ce qu'elle implique dans chaque cas, chaque site, chaque collection, il faut non seulement avoir du Pist une vision complète, cohérente et, surtout, la plus claire possible, mais aussi des outils de connaissance multiples et affûtés.

10 À partir du socle de la connaissance historique et de la méthode scientifique d'analyse de la «trace matérielle» de l'activité économique, scientifique et technique, la formation développe la polyvalence nécessaire aux deux capacités d'expertise, celles de définition et de conduite de projet. Les étudiants doivent apprendre à maîtriser un environnement en évolution et s'initier à nombre de spécialités pour être en mesure d'élaborer et de conduire des projets de valorisation et de gestion, tant dans le domaine public que privé. Ils sont mis en contact avec la réalité des objets et des sites, on leur fait saisir les mécanismes qui expliquent la reconnaissance, la préservation, la conservation et l'interprétation du patrimoine, ou son délaissement, en des phases parfois alternées sur un moyen ou un long terme. Un bon professionnel doit pouvoir analyser le jeu des acteurs à l'œuvre en un lieu ou sur un projet et harmoniser les temporalités propres à chacun : annualité budgétaire d'une administration, mandat d'une assemblée, délai fixé par l'entreprise au retour sur investissement, temps nécessaire pour la recherche et pour la décision au regard du postulat d'une transmission patrimoniale sans fin. En particulier, les étudiants sont invités à ne pas ou plus considérer les entreprises uniquement comme productrices lointaines de patrimoine industriel, ni comme interlocutrices impossibles ou absentes. À charge pour eux de prendre conscience de la chaîne qui va de l'activité économique à la valeur patrimoniale, de favoriser des rapprochements heureux (et non des confusions, sinon des collusions malheureuses) entre les besoins de l'entreprise en matière de circulation des savoirs et des informations ainsi que de communication interne et externe avec la préservation et la transmission $d u$ patrimoine technique et industriel. Ce développement élargit le champ du Pist et, de ce fait, l'intervention professionnelle audelà du patrimoine « patrimonialisé » par la loi ou par l'institution culturelle.

11 La formation est organisée en cinq domaines représentant un ensemble de plus de 450 heures de cours, complété par un stage de seize semaines au moins. L'enseignement, délivré en début d'année, est assuré aux deux tiers par des professionnels. Outre les enseignements qu'ils dispensent dans leur domaine de spécialité (par exemple le management de projet, la communication, le tourisme de découverte économique), leur témoignage doit amener les étudiants à ordonner les différentes matières, informations, rencontres dans leur propre définition du Pist, celle qui leur servira de référence dans leur activité, qu'ils élaborent à partir d'un panorama historique et professionnel du patrimoine étudié. Les étudiants sont formés en majorité par les études d'histoire mais viennent aussi de l'histoire de l'art, de l'ethnologie, du tourisme. Pour permettre aux diplômés qu'ils seront d'être en mesure d'établir un diagnostic global d'un site patrimonial, du point de vue de son histoire, de sa valeur et de ses possibilités de développement, et de définir les axes appropriés d'un programme de sauvegarde ou de mise en valeur, leur apprentissage comprend le management de projets et d'équipes, l'analyse des marchés et des publics, la connaissance du droit administratif, du droit de l'urbanisme, de la fonction publique territoriale et de la politique locale. L'intégration de la formation dans le master "Construction et dynamiques des espaces, des sociétés et des économies» de la faculté d'histoire/ géographie a renforcé ces aspects, avec l'apparition d'un tronc commun aux différentes 
spécialités professionnelles, Pist et «Environnement, développement, territoires" (alors que les DESS étaient indépendants les uns des autres) comportant les enseignements de gestion et marketing, communication, informatique, «insertion professionnelle » (préparation des concours administratifs). L'enseignement propre à la spécialité Pist comprend "la connaissance du Pist» (trois enseignements, dont l'histoire de l'industrie et une nouvelle formation spécifique, les archives du Pist, avec de nombreux travaux pratiques dans les centres d'archives de la région) et la " conduite et management d'un projet de Pist» (dont le management de projet, la muséologie et le droit).

En outre, de nombreux spécialistes interviennent de manière ponctuelle: représentants de l'administration centrale de la Culture, conservateurs de musées techniques, acteurs économiques, chefs de services patrimoniaux dans les entreprises, architectes et enseignants en architecture, responsables d'associations nationales et locales de préservation et d'animation du patrimoine, universitaires, ils dispensent un enseignement précis, de la médiation à l'édition scientifique en passant par la muséographie ou l'ingénierie scientifique et culturelle, tout en initiant les étudiants à leur domaine d'activité, contribuant ainsi à les orienter dans le choix de leur stage professionnel, puis dans leur recherche d'emploi.

Des visites sur le terrain enrichissent cette formation (archives départementales du Pas-de-Calais, Camt de Roubaix, cristallerie d'Arques, distillerie Persyn à Houlle, ascenseur à bateaux des Fontinettes, Familistère Godin à Guise, Cnam/Musée des arts et métiers) et les étudiants sont incités à participer à des journées et à des colloques ${ }^{4}$.

Le stage, enfin, est un temps essentiel. Les propositions d'accueil sont largement supérieures au nombre d'étudiants. Elles proviennent de toute la France - pas seulement de la région - et même de l'étranger (Canada en 2005), d'administrations de la Culture, d'établissements culturels dépendant ou non de collectivités et installés sur des sites de patrimoine industriel reconvertis, d'entreprises (ou de leur fondation) et de structures les fédérant. Deux mémoires, un rapport de stage et un projet professionnel, sanctionnent cette mise en situation.

Enfin, pour contribuer au développement du Pist comme domaine d'activité, promouvoir la coopération des acteurs économiques et scientifiques et ouvrir aux étudiants de nouveaux chantiers, son enseignement sera lié à la "recherche-action " par la promotion de projets de recherche alliant des opérations d'inventaire et de mises en valeur. Un projet a été élaboré en juin 2005. Il a pour objectif la définition, l'étude et la sauvegarde de traces matérielles et immatérielles de l'activité économique témoignant de l'histoire des réseaux de transport et communication en Europe qui, à la différence des nœuds de réseaux, ont été jusqu'ici négligées tant par les recherches historiques que par les actions de préservation du patrimoine.

Depuis son ouverture, la formation attire des candidatures venant de France et de l'étranger. Elles émanent surtout d'étudiants en quête d'une voie professionnalisante et, trop rarement encore, de personnels d'entreprises ou d'administration qui bénéficient plus ou moins aisément des possibilités offertes par la formation continue. Les questions que pose l'adéquation de l'offre à la demande sont multiples. Il est difficile d'évaluer la pertinence d'une formation après seulement deux années complètes de fonctionnement. Une enquête récente a cependant montré, d'une part, que l'ensemble des diplômés étaient employés, les contrats à durée indéterminée remplaçant les contrats précaires après deux années en moyenne d'activité; d'autre 
part, elle a révélé la prédominance des employeurs publics, collectivités publiques, associations dépendant d'elles et établissements publics à vocation culturelle. Seul le concours spécialisé de la fonction publique semble, pour les étudiants, garantir la durée de l'emploi et est explicitement réclamé par ce type d'employeur. On peut le regretter, car le développement du secteur "patrimoine" aux franges et en dehors du secteur public encourage à la création de formations professionnelles polyvalentes qui ne répondent pas à ces spécialités ou en dépassent les limites.

Le patrimoine industriel, scientifique et technique est en mutation, qu'il s'agisse de sa définition, de son appropriation par l'ensemble des acteurs et des publics comme objet politique, économique, culturel, social, ou des conséquences de cette mutation sur son existence comme domaine d'activité professionnelle.

Depuis 2002, le master 2 professionnel Pist de l'université d'Artois y prend sa part, à l'écoute des étudiants, des intervenants et des diplômés, attentif aux possibilités d'emplois, aux progrès et à l'actualité de la recherche. Il s'efforce de maintenir un lien réel entre recherche et enseignement, enseignement et projet, projet et recherche et un dialogue actif entre les acteurs, à l'échelle de la région dans laquelle il est implanté mais aussi en favorisant des échanges en Europe et au-delà.

Cependant, plus de trente ans après avoir été imposé par la communauté scientifique et reconnu par la puissance publique, le Pist doit encore être perçu comme un domaine de compétences professionnelles spécifiques. Il lui faut construire sa "filière » chez les différents acteurs publics et privés qui font le monde professionnel d'un champ dans lequel ses diplômés ont la volonté, par-delà les obstacles, d'exercer leur ministère.

Un itinéraire

Candidate au DESS (première promotion, 2002-2003) avec une maîtrise d'histoire contemporaine et déjà une expérience (temps partiel) de travail dans une commune, cette étudiante a effectué son stage professionnel dans une association liée à cette même commune du bassin minier, qui a pour mission de rassembler les éléments de la mémoire locale et de les promouvoir, en mettant à la disposition du public une documentation spécifique. Elle est restée une année après son diplôme dans cette municipalité, pendant laquelle elle a poursuivi parallèlement un master 2 Recherche sur le patrimoine du bassin minier. Employée ensuite par une autre commune, Billy-Montigny, elle a été chargée de la mise en œuvre du projet de Centre d'interprétation des risques industriels (du chantier du bâtiment à la réunion des collections et au pilotage des multiples manifestations commémorant en 2006 la catastrophe de Courrières, dont deux colloques internationaux). Pour sa troisième année d'activité, elle a été recrutée par la communauté d'agglomération Lens-Liévin pour mettre en valeur la maison syndicale, récemment classée monument historique, en traiter les fonds d'archives et proposer un projet culturel et scientifique de mise en valeur de ce patrimoine social du bassin minier (expositions, conférences débats, gestion du lieu, partenariats et diffusion des activités). Son statut a évolué de "agent du patrimoine» (10 h/semaine) en CDD dans la première municipalité à « chargée de mission » en CDD de trois ans dans la seconde, interrompu après un an pour un CDD de trois ans, susceptible de devenir CDI à la communauté d'agglomération Lens-Liévin. 


\section{NOTES}

1.Pour des renseignements pratiques, consulter le site : www.univartois.fr/francais/ formation/ufr/histgeo/formation/master2pist.htm

2.Industrieel erfgoed in Noord-Frankrijk (Patrimoine industriel du Nord de la France), numéro spécial de Erfgoed van Industrie en Techniek - Vlaams-Nederlands Tijdschrift (Patrimoine de l'industrie et des techniques, Flandres et Pays-Bas), $3^{\mathrm{e}}$ trimestre 2004 ; Olivier Kourchid, Hélène Melin, « Mobilisations et mémoire du travail dans une grande région : le Nord - Pas-de-Calais et son patrimoine industriel ", Le Mouvement social, $\mathrm{n}^{\circ}$ 199, avril-juin 2002, p. 37-59.

3.Pour se faire une idée de cette offre, consulter le site : http://www.visiteentreprise.com

4.Salon du patrimoine culturel, Paris, Carrousel du Louvre, novembre 2002 ; "L'industriel face à son patrimoine historique ", Paris, Patrimoine et mémoire d'entreprises, novembre 2002 ; « Industrie et culture », Lewarde, AMSCTI, juillet 2003 ; «La reconversion des bassins charbonniers. Une comparaison interrégionale entre Nord - Pas-de-Calais et Ruhr », Lille-Roubaix, novembre 2003 ; « La mémoire de l'industrie : de l'usine au patrimoine ", Besançon, MSH de Franche-Comté, novembre 2003 ; CILAC 2004, « 30 ans de patrimoine industriel en France », Le Creusot, université de Bourgogne, septembre 2004 ; Conservare 2005-Forum européen du Patrimoine, Courtrai, 7-10 octobre 2005.

\section{RÉSUMÉS}

Le patrimoine correspond désormais à un secteur professionnel à part entière. L'université d'Artois l'a développé dans ses offres de formation en proposant le master 2 professionnel Pist (Patrimoine industriel, scientifique et technique). L'article présente, après trois années d'expérience, son champ d'action, ses orientations et ses contenus avec les développements escomptés dans le domaine de la "recherche-action", enfin ses débouchés. Il souligne le décalage existant entre la récente légitimation du patrimoine industriel comme objet scientifique, culturel, économique et l'émergence d'une nouvelle filière professionnelle consacrée à sa connaissance, sa gestion, sa mise en valeur et son interprétation.

\section{INDEX}

Mots-clés : université d'Artois, master 2 professionnel Pist (Patrimoine industriel, scientifique et technique), patrimoine industriel, scientifique et technique 


\section{AUTEURS}

\section{MARIE-NOËLLE POLINO}

Marie-Noëlle Polino, ancienne élève de l'École normale supérieure (Sèvres), est secrétaire générale et scientifique de l'Association pour l'histoire des chemins de fer en France (AHICF) ainsi que secrétaire générale de Patrimoine et mémoire d'entreprises, association des entreprises pour la sauvegarde et la mise en valeur du patrimoine technique et industriel. Elle est responsable de deux modules de formation du master Pist de l'université d'Artois.

\section{DENIS VARASCHIN}

Denis Varaschin est professeur d'histoire contemporaine à l'université d'Artois. Il a fondé la formation et en a assuré la responsabilité de septembre 2002 à 2005. Directeur de l'EA 2466 et du « pôle Artois » de la MSH Nord-Pas-de-Calais, il est spécialisé en histoire des entreprises, de l'industrie et des techniques. 